\title{
Palisaded encapsulated neuroma (solitary circumscribed neuroma of skin) of the eyelid: report of two cases and review of the literature
}

\author{
Sander R Dubovy, Brian J Clark
}

\begin{abstract}
Backgroundlaims-Palisaded encapsulated neuroma of the skin (PEN) is an uncommon benign neural tumour that occurs on the head and neck, including the eyelid, but is unfamiliar to many ophthalmic clinicians. For the first time in the ophthalmic literature, the clinical and histopathological features of two patients with PEN of the eyelid are described.

Methods-Two cases of PEN were identified in the archives of the Department of Pathology, Institute of Ophthalmology, London. Clinical details were derived by a case note review and the histopathological features defined using standard methods including immunohistochemistry.

Results-Two cases of PEN are described, from the eyelids of patients aged 43 and 70 years. The tumours were virtually identical in nature, being painless solid masses composed of a mixture of $\mathrm{S} 100$ positive Schwann cells and scattered neurofilament positive nerve fibres. An incomplete thin fibrous capsule, which contains EMA positive perineural cells, partially surrounded each lesion. The lesions were distinguishable from other more common eyelid lesions only on histopathological examination. Both tumours were completely excised with no evidence of recurrence. Neither patient had signs of any associated systemic disease.

Conclusion-PENs are usually solitary, firm, non-pigmented, dome-shaped lesions that are found on the face of patients usually over the age of 40 years. Most often these lesions are diagnosed clinically as basal cell carcinomas, dermal naevi, or skin adnexal tumours. Histologically, PENs must be differentiated from other neural tumours that may be associated with systemic syndromes, such as neurofibromas or mucosal neuromas.

(Br f Ophthalmol 2001;85:949-951)
\end{abstract}

In 1972 Reed and colleagues first described 44 cases of a distinctive neural tumour, which they named palisaded, encapsulated neuroma of the skin. They described solitary, firm, nonpigmented, dome-shaped nodules that were primarily located on the face of patients between the age of 40 and 60 years. In most cases the clinical diagnosis was dermal naevus or basal cell epithelioma. Since that report a few series describing this uncommon lesion have been published, ${ }^{1-10}$ and two have described them occurring in the eyelid. ${ }^{13}$ However, to date there have been no reports of this entity in the ophthalmic literature and, as a consequence, ophthalmologists and some ophthalmic pathologists may be unaware of this rare entity. Here, we report the clinicopathological findings of two cases of palisaded, encapsulated neuroma of skin occurring in the eyelid.

\section{Materials and methods}

Two cases of PEN were identified from the archives of the Department of Pathology, Institute of Ophthalmology, London. Clinical details were obtained by case note review and the histopathological features of each case defined by standard methods using haematoxylin and eosin staining and immunohistochemistry for S100 protein (Dako), antineurofilament (Dako) and anti-epithelial membrane (Dako) antibodies.

\section{Results}

CLINICAL FEATURES

Case 1

A healthy 43 year old woman with a history of bilateral pterygium was found to have a "papillomatous left lower lid lump" measuring $3 \mathrm{~mm}$ in diameter. The lesion was painless, firm, and had been slowly growing for many years. The patient underwent excision of the pterygium in the right eye and excisional biopsy of the lid lesion in the left eye. She tolerated the procedure well and has had no recurrences 11 months after the operation. There are no signs of any associated systemic disease.

\section{Case 2}

A healthy 70 year old man was noted on examination to have a "cystic lesion" of the right upper eyelid which measured $6 \mathrm{~mm}$ in greatest diameter. The painless lesion had been present for a number of years, was firm to palpation, with no eyelashes growing through it. The mass was excised and no recurrences have been reported 13 months postoperatively. He has no signs of any associated systemic disease.

\section{HISTOPATHOLOGY}

Sections of both cases showed very similar features. Each lesion consisted of rounded superficial dermal tumours that occupied the bulk of the biopsies (Fig 1A). Despite the clinical descriptions of "papillomatous" and "cystic," both lesions were solid and nodular. The lesions were partly surrounded by a thin capsule of collagen and staining with epithelial 


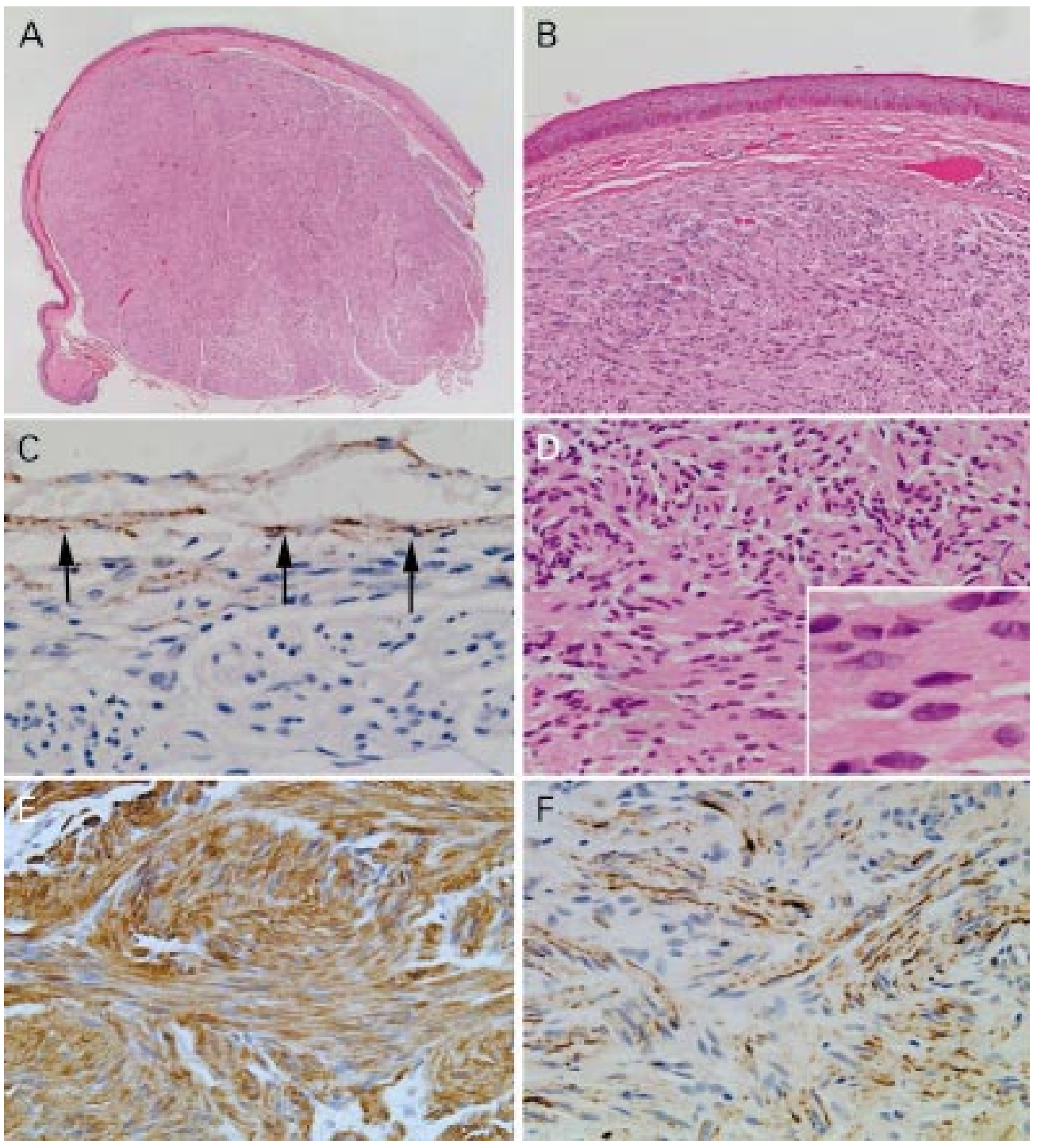

Figure 1 (A) A rounded, superficial dermal lesion occupies the majority of the biopsy (haematoxylin and eosin, original magnification $\times 1.25$ ). (B) The lesion is well circumscribed and partly surrounded by a thin capsule of collagen (haematoxylin and eosin, original magnification $\times 10$ ). (C) The cells within the capsule stain positively (arrows) for epithelial membrane antigen (streptavidin-biotin immunoperoxidase-DAB, original magnification $\times 40$ ). (D) The lesion consists of numerous tightly packed fascicles of spindle-shaped cells with eosinophilic cytoplasm and indistinct cell borders (haematoxylin and eosin, original magnification $\times 20$ ); inset (haematoxylin and eosin, original magnification $\times 60)$. (E) The cells stain intensely for $S 100$ protein (streptavidin-biotin immunoperoxidase-DAB, original magnification $\times 20)$. $(F)$ Immunohistochemical staining with neurofilament protein discloses numerous axons present throughout the lesion (streptavidin-biotin immunoperoxidase-DAB, original magnification $\times 20$ ).

membrane antigen (EMA) denoted the presence of perineural cells within the capsule (Fig $1 \mathrm{~A}-\mathrm{C})$. The lesions consisted of numerous tightly packed fascicles of Schwann cells that were spindle-shaped, had eosinophilic cytoplasm with indistinct cell borders (Fig 1D), and stained intensely for S100 protein (Fig $1 E)$. Immunohistochemical staining with neurofilament protein disclosed numerous axons present throughout the lesions (Fig $1 F$ ). No evidence of pleomorphism or mitotic activity was identified.

\section{Discussion}

Palisaded encapsulated neuroma of skin, also known as solitary circumscribed neuroma, is a clinically distinctive, solitary, benign cutaneous tumour that is most often found on the face near the margin of a mucocutaneous junction. ${ }^{1-10}$ These lesions have also been described on the trunk, shoulder, arm, hand, foot, oral mucosa, nasal fossa, and glans penis. ${ }^{1-10}$ The majority of patients are between 30 and 60 years of age and note slow progression of the tumour over a number of years. ${ }^{1-3810}$ The lesions usually measure between 2 and $6 \mathrm{~mm}$, are solitary, sessile, firm, pink, or flesh coloured, dome-shaped, with loss of overlying skin markings. No hairs are usually seen growing from their surface. ${ }^{1-10}$ Telangiectasia is usually minimal or absent and ulceration is only present after secondary trauma. ${ }^{1-38}$ Clinically they are most frequently diagnosed as basal cell carcinoma, melanocytic naevus, epidermal cyst, or skin appendage tumours. ${ }^{1-35} 56$ The long duration of tumour, sparsity of telangiectasia, lack of ulceration, and circumscription may help to differentiate the PEN from a basal cell carcinoma. Intradermal naevi tend to be softer than these 
lesions and usually retain the overlying skin markings. Small appendage tumours such as eccrine spiradenoma are very difficult to differentiate clinically from the palisaded encapsulated neuroma..$^{1-356}$

Macroscopically the lesions are usually firm, well circumscribed grey-white intradermal nodules. ${ }^{3}$ Histologically, the PEN is a well circumscribed, partially encapsulated spindle cell tumour. It consists of broad, interlacing, elongated fascicles of bland spindle cells. The fascicles appear distinct from one another and are often separated by artefactual clefts. The tumour cells are poorly delineated with eosinophilic cytoplasm, and pointed, wavy basophilic nuclei. No pleomorphism is seen and mitotic figures are rarely present. ${ }^{2}{ }^{2}$ Occasional small blood vessels are seen in the stroma but haemorrhage and necrosis have not been reported. ${ }^{38}$ The tumour cells are positive for S100 protein, collagen type IV, and vimentin, all of which are consistent with Schwann cell differentiation. The capsule contains flattened, elongated perineural cells that stain positively for EMA. A variable number of small axons are identified within the fascicles on staining with neurofilament antibodies. ${ }^{3-10}$ The nodular type is seen most frequently with other less common variants reported: epithelioid, plexiform, multinodular, increased vasculature, and a fungating growth pattern. ${ }^{10}$

The lesion must be differentiated histologically from the neurofibroma, schwannoma, traumatic neuroma, and leiomyoma. ${ }^{1-36810}$ The neurofibroma, which may be associated with von-Recklinghausen's disease, lacks a capsule, contains mucopolysaccharide ground substance, and has fewer axons with myelin sheaths. The schwannoma usually is present in a subcutaneous location, contains Antoni A and B type tissue with Verocay bodies, and lacks axons. The traumatic neuroma contains axonal and Schwann cell proliferation with the addition of scarring and inflammatory cells in contradistinction to PEN. The leiomyoma is a spindle cell lesion composed of muscle cells that can be readily differentiated by immunohistochemical stains. ${ }^{1-36810}$

The histiogenesis of this lesion has been postulated to be a primary hyperplasia of the nerve fibres. Since axons cannot duplicate, but do regrow, the PEN may be a hamartomatous growth in which there is a rough duplication of the normal Schwann cell to axon relation. The cause of this regrowth is unknown. Some have speculated that there is a traumatic origin; however, histological methods have not supported this. $^{25-7}$

The importance of correctly diagnosing these tumours is that they are benign and rarely recur. Misdiagnosis as a neurofibroma or mucosal neuroma may lead to unnecessary clinical concern and patient testing. The diagnosis of neurofibroma causes the clinician to examine for evidence of von Recklinghausen's disease and, with it, the concern of a malignant peripheral nerve sheath tumour arising from a neurofibroma. Mucosal neuromas are seen in type IIb multiple endocrine neoplasia syndrome, Gorlin syndrome, and bring the concern of accompanying occult medullary carcinoma of the thyroid and phaeochromocytoma. Palisaded encapsulated neuromas are usually present in the skin near a mucocutaneous junction and in almost all cases are single as opposed to the multiple mucosal neuromas seen in the MEN syndromes. ${ }^{3} 6910$

This work received a proportion of its funding from the NHS Executive; the views expressed in this publication are those of the authors and not necessarily those of the NHS Executive. We are grateful to Miss R Hart and Miss D Hornall for their technical expertise. These cases have been presented at the meeting of the British Association for Ophthalmic Pathology, Dublin 2000 and the Combined Meeting of the International Society of 2000 and the Combined Meeting of the International Society of
Ophthalmic Pathology and the American Association of Ophthalmic Pathology and the Am
Ophthalmic Pathologists, Dallas 2000.

1 Reed RJ, Fine RM, Meltzer HD. Palisaded, encapsulated neuromas of the skin. Arch Dermatol 1972;106:865-70.

2 Dover JS, From L, Lewis A. Palisaded encapsulated neuromas. Arch Dermatol 1989;125:386-9.

3 Fletcher CDM. Solitary circumscribed neuroma of the skin. Am f Surg Pathol 1989;13:574-80.

4 Albrecht S, Kahn HJ, From L. Palisaded encapsulated neuroma: an immunohistochemical study. Mod Pathol 1989;2:403-6

5 Argenyi ZB. Immunohistochemical characterization of palisaded, encapsulated neuroma. I Cutan Pathol 1990;17: 329-35.

6 Argenyi ZB. Newly recognized neural neoplasms relevant to the dermatopathologist. Dermatol Clin 1992;10:219-34.

7 Dakin MC, Leppard B, Theaker JM. The palisaded, encapsulated neuroma (solitary circumscribed neuroma). Histopathology 1992;20:405-10.

8 Megahed M. Palisaded encapsulated neuroma (solitary circumscribed neuroma). A clinicopathologic and immunohistochemical study. Am f Dermatopath 1994;16:120-5.

9 Val-Bernal JF, Alvarez-Canas C, Vega A. Palisaded encapsulated neuroma of the glans penis. Urology 1995;45:689-91.

10 Mayorga M, Acebo E, Val-Bernal JF. Palisaded encapsulated neuroma of the nasal fossa. Otolaryngol Head Neck Surg 1998;119:141-3. 